Tôhoku Math. Journ.

Vol. 19, No. 4,1967

\title{
SELF-INJECTIVE RINGS
}

\author{
TOYONORI KATO
}

(Received July 25, 1967)

Throughout this paper each ring $R$ will be a ring with identity element and each module will be unital. We denote the category of right (resp. left) $R$-modules by $\mathfrak{M}_{R}\left(\right.$ resp. $\left.{ }_{R} \mathfrak{M}\right)$.

If $A$ is a module over a ring $R$, we denote the dual of $A$ by $A^{*}$. We may form the double dual $A^{* *}$ of $A$ and get the natural $R$-homomorphism

$$
\delta_{A}: A \longrightarrow A^{* * *} .
$$

Following H.Bass [2, p. 476], we call $A$ torsionless if $\delta_{A}$ is a monomorphism and reflexive if $\delta_{A}$ is an isomorphism. Let $A$ be a right $R$-module. If $X$ is a subset of $A$, then we set

$$
l(X)=\left\{b \in A^{*} \mid b X=0\right\} .
$$

Similarly, if $X$ is a subset of $A^{*}$, then we set

$$
r(X)=\{a \in A \mid X a=0\} .
$$

Note that, if $X$ is a subset of a ring $R, l(X)$ (resp. $r(X)$ ) is just the left (resp. right) annihilator of $X$ in $R$ since $\left(R_{R}\right)^{*}={ }_{R} R$. We call a ring $R$ right $\mathrm{PF}$ if every faithful right $R$-module is a generator in $\mathfrak{M}_{R} . A$ ring $R$ is $\mathrm{PF}$ if $R$ is both right and left PF. A ring which is both right and left self-injective is called self-injective.

In the present paper we shall show the following theorems :

THEOREM 7. The following conditions on a ring $R$ are equivalent:

(1) $R$ is right $\mathrm{PF}$.

(2) $R$ is right self-injective and $l(I) \neq 0$ for each maximal right ideal $I$.

(3) $R$ is an injective cogenerator in $\mathfrak{M}_{R}$.

THEOREM 8. The following conditions on a ring $R$ are equivalent:

(1) Every submodule and every quotient module of a reflexive right $R$-module is reflexive and every submodule and every quotient module of a reflexive left $R$-module is also reflexive. 
(2) Every finitely generated right, and every finitely generated left, $R$ module is reflexive.

(3) Every cyclic right, and every cyclic left, $R$-module is reflexive.

(4) $R$ is PF.

(5) $R$ is self-injective and $l(I) \neq 0$ for each maximal right ideal $I$.

(6) $R$ is an injective cogenerator both in $\mathfrak{M}_{R}$ and in ${ }_{R} \mathfrak{M}$.

THEOREM 9. Let $R$ be a ring characterized in Theorem 8, and $A$ a right $R$-module. Then

(1) $r\left(l\left(A_{0}\right)\right)=A_{0}$ for every submodule $A_{0}$ of $A$.

(2) If $A$ is finitely generated (or reflexive), then $l\left(r\left(B_{0}\right)\right)=B_{0}$ for every submodule $B_{0}$ of $A^{*}$.

THEOREM 10. The following conditions on a ring $R$ are equivalent:

(1) $R$ is $\mathrm{PF}$ and the Jacobson radical of $R$ is nilpotent.

(2) $R$ is QF.

1. Cogenerators. In this section we study rings $R$ for which $R$ is an injective cogenerator in $\mathfrak{M}_{R}$ and deduce the interesting characterizations of such rings. The following proposition paves the way for the definition of cogenrators in $\mathfrak{M}_{R}$.

PROPOSITION 1. The following conditions on right $R$-modules $A$ and $C$ are equivalent: of $C$.

(1) There is a monomorphism from $A$ into a direct product of copies

(2) For any nonzero map $B \rightarrow A\left(B \in \mathfrak{M}_{R}\right)$, there is a map $A \rightarrow C$ such that the composition map $B \rightarrow A \rightarrow C$ is nonzero.

(3) For any nonzero element a of $A$, there is a map $f: A \rightarrow C$ such that $f(a) \neq 0$.

This result is clear and we omit the proof.

A right $R$-module $C$ is called a cogenerator in $\mathfrak{M}_{R}$ if it satisfies the conditions in Proposition 1 for each right $R$-module $A$. Note that a ring $R$ is a cogenerator in $\mathfrak{M}_{R}$ if and only if every right $R$-module is torsionless.

If $A$ is a right $R$-module then $E(A)$ will denote the injective hull of $A$, and let $B^{\prime} \supset A$ signify that a right $R$-module $B$ is an essential extension of A.

The following proposition is a useful characterization of being a cogener. ator in $\mathfrak{M}_{R}$.

PROpOSITION 2. A right $R$-module $C$ is a cogenerator in $\mathfrak{M}_{R}$ if and only if $C$ contains a copy of the injective hull $E(U)$ of each simple right $R$-module $U$. 
PROOF. Let $C$ be a cogenerator in $\mathfrak{M}_{k}$, and $U$ a simple right $R$-module. By Proposition 1 we have a map $g: E(U) \rightarrow C$ such that $U \rightarrow E(U) \rightarrow C$ is nonzero where $U \rightarrow E(U)$ is inclusion. The map $g$ is a monomorphism since $\operatorname{Ker}(g) \cap U=0$ and $E(U)^{\prime} \supset U$. Conversely let $C$ contain a copy of $E(U)$ for each simple right $R$-module $U$. Let $A$ be any right $R$-module, and $a \neq 0 \in A$. Then there is a map $f: a R \rightarrow E(U)$ such that $f(a) \neq 0$ for some simple right $R$-module $U$. The map $f$ can be extended to a map $f^{\prime}$ of $A$ into $E(U)$ since $E(U)$ is injective. The map $f^{\prime}$ is considered as a map of $A$ into $C$ by assumption and $f^{\prime}(a) \neq 0$, which completes the proof.

The following theorem will be of some interest.

THEOREM 1. The following conditions on a right self-injective ring $R$ are equivalent:

(1) $\left.r^{\prime} l(I)\right)=I$ for each right ideal $I$.

(2) $l(I) \neq 0$ for each maximal right ideal $I$.

(3) $R$ is a cogenerator in $\mathfrak{M}_{R}$.

ProOF. The implication $(1) \Rightarrow(2)$ is trivial.

(2) implies (3). Let $U$ be a simple right $R$-module. Then $U \approx R / I$ for some maximal right ideal $I$. Then we have

$$
U^{*} \approx(R / I)^{*} \approx l(I) \neq 0 .
$$

Hence $R$ contains a copy of $U$. Thus $R$ is a cogenerator in $\mathfrak{M}_{R}$ since $R$ is right self-injective.

(3) implies (1). Since $R$ is a cogenerator in $\mathfrak{M}_{R}$, every cyclic right $R$ module is torsionless. Thus $\left.r^{\prime} l(I)\right)=I$ for each right ideal $I$ by [6, Theorem 1].

We denote by $J$ the Jacobson radical of a $\operatorname{ring} R$.

On the rings characterized in the preceding theorem, B.L.Osofsky [8] has proved the following surprising result.

THEOREM 2. Let $R$ be an injective cogenerator in $\mathfrak{M}_{R}$. Then $R / J$ is Artinian.

REMARK. It is easy to see that, if $R$ is an injective cogenerator in $\mathfrak{M}_{R}$ with zero Jacobson radical, then $R$ is Artinian. In fact, $R$ does not contain any proper essential right ideal since $r(l(I))=I$ for each right ideal $I$ and $J=\left\{x \in R \mid R^{\prime} \supset r(x)\right\}=0$ (see [3, Theorem 3.1] or [9, Lemma 4.1]).

Note that, if $R$ is right self-injective, $R / J$ is Artinian if and only if every simple right $R$-module is isomorphic to $e R / e J$ for some idempotent $e \in R$. The 'only if' part is evident since idempotents can be lifted modulo $J$ (see [7, Proposition 4] or [9, Corollary 3.2]). The 'if' part follows since $R / J$ 
is an injective cogenerator in $\mathfrak{M}_{R / J}$ (see [9, Theorem 4.8]).

Recall the definition of generators in $\mathfrak{M}_{R}$. A right $R$-module $M$ is a generator in $\mathfrak{M}_{R}$ if each right $R$-module is an epimorph of a direct sum of copies of $M$. Note that $M$ is a generator in $\mathfrak{M}_{R}$ if and only if $R$ is a direct summand of a finite direct sum of copies of $M$.

PROposition 3. A projective right $R$-module $M$ is a generator in $\mathfrak{M}_{R}$ if and only if each simple right $R$-module is an epimorph of $M$.

Proof. Let $M$ be a generator in $\mathfrak{M}_{R}, U$ a simple right $R$-module. Then we can find easily a nonzero map $M \rightarrow U$, which is necessarily an epimorphism since $U$ is simple. Conversely, let us suppose that each simple right $R$-module is an epimorph of $M$. Let $I$ be a maximal right ideal of $R$. Then there exists a map $f: M \rightarrow R$ such that the composition map $M \rightarrow R \rightarrow R / I$ is nonzero, or equivalently, $f(M) \not \subset I$ since $M$ is projective. Hence $R$ is a sum of those right ideals which are epimorph of $M$, which completes the proof.

Now, let $\left\{U_{i}\right\}$ be the family of all non-isomorphic simple right $R$-modules, and $C$ the direct sum of the family $\left\{E\left(U_{i}\right)\right\}$.

THEOREM 3. The following conditions on a ring $R$ are equivalent:

(1) $R$ is an injective cogenerator in $\mathfrak{M}_{R}$.

(2) $C$ is a generator in $\mathfrak{M}_{R}$.

Proof. (1) implies (2). $E\left(U_{i}\right)$ is projective, or equivalently, $C$ is projective since $R$ is a cogenerator in $\mathfrak{M}_{R}$. Each simple right $R$-module is isomorphic to $E\left(U_{i}\right) / E\left(U_{i}\right) J$ for some simple right $R$-module $U_{i}$ since $R / J$ is Artinian (see [4, p. 214]). Thus each simple right $R$-module is an epimorph of $C$ and hence $C$ is a generator in $\mathfrak{M}_{R}$ by Proposition 3 .

(2) implies (1). Since $C$ is a generator in $\mathfrak{M}_{R}, R$ is right self-injective and a finite direct sum of indecomposable right ideals each of which contains a minimal right ideal of $R$ (see [1, p. 702]). Then $R / J$ is Artinian. Hence $R$ contains a copy of each simple right $R$-module, thus completing the proof.

2. Simple modules. In this section we shall consider simple modules over rings characterized in Theorem 1 and obtain a precise information on the structure of these rings.

Let $R$ be a right self-injective ring, $L$ a finitely generated left ideal of $R$. Then $l(r(L))=L$ (see [5] or [6, Theorem 13]). We shall use this fact to show the following.

LEMMA 1. Let $R$ be a right self-injective ring satisfying the conditions in Theorem 1 . Let $I$ be a maximal right ideal of $R$. Then $l(I)$ is a minimal left ideal of $R$. 
PROOF. Let $a \neq 0 \in l(I)$; then we have

$$
\left.I \subset r^{\prime} l(I)\right) \subset r^{\prime}(a) \neq R
$$

Then $I=r^{\prime}(a)$ since $I$ is maximal. Hence we have

$$
l(I)=l\left(r^{\prime}(a)\right)=R a
$$

which completes the proof.

THEOREM 4. Let $R$ be a right self-injective ring satisfying the conditions in Theorem 1. Then the mapping

$$
U \longrightarrow U^{*}
$$

gives a one-to-one correspondence between isomorphism classes of simple right $R$-modules and isomorphism classes of simp'e left $R$-modules.

Proof. Let $U$ be a simple right $R$-module. Then $U \approx R / I$ for some maximal right ideal $I$. $U^{*} \approx l(I)$ is simple by Lemma 1 . Now, we may assume that $U$ is a minimal right ideal of $R$ since $R$ is a cogenerator in $\mathfrak{M}_{R}$. Then $U$ is reflexive since $R$ is right self-injective and $r(l(U))=U$ (see [6, Proposition $5])$. This implies that our correspondence is one-to-one. Finally, our correspondence is 'onto' since $R / J$ is Artinian.

Let $R$ be a right self-injective ring characterized in Theorem 1 . In the proof of Theorem 4 we have just seen that each simple right $R$-module is reflexive. Hence each simple left $R$-module is also reflexive by Theorem 4 .

Y. Utumi [10, Theorem 1.3] has proved the following interesting result on the Jacobson radical $J$ of a right self-injective ring.

THEOREM 5. Let $R$ be a right self-injective ring, and I a right (or left) ideal of $R$. Then $I \subset J$ if and only if $I$ does not contain any nonzero idempotent.

THEOREM 6. Let $R$ be a right self-injective ring characterized in Theorem 1.

(1) $r^{\prime}(L)$ is a minimal right ideal for each maximal left ideal $L$.

(2) er $(J)$ (resp. $l(J) e)$ is a minimal right (resp. left) ideal for every primitive idempotent $e$, and each simple right (resp. left) $R$-module is isomorphic to er $(J)$ (resp. $l(J) e)$ for some primitive idempotent e.

(3) $l(J)=r^{\prime}(J)$ and $l\left(r^{\prime}(J)\right)=J$.

(4) Every nonzero right (resp. left) ideal contains a minimal right (resp. left) idcal. 
PROOF. (1) Let $L$ be a maximal left ideal of $R$. Then $r(L) \approx(R / L)^{*}$ is simple by Theorem 4 .

(2) Let $e$ be a primitive idempotent. Then $(1-e) R+e J($ resp. $R(1-e)+J e)$ is a maximal right ideal (resp. left ideal). Hence $\operatorname{er}(J)=r(R(1-e)+J e)$ (resp. $l(J) e=l((1-e) R+e J))$ is minimal by (1) (resp. by Lemma 1$)$. Note that each simple right (resp. left) $R$-module is isomorphic to $e R / e J$ (resp. $R e / J e$ ) for some primitive idempotent $e$. Since $(e R / e J)^{*} \approx l(J) e,(R e / J e)^{*}=\operatorname{er}(J)$, each simple right (resp. left) $R$-module is isomorphic to $\operatorname{er}(J)$ (resp. $l(J) e$ ) by Theorem 4.

(3) $\operatorname{er}(J) \subset l(J)$ (resp. $l(J) e \subset r(J)$ ) for every primitive idempotent $e$ by (2). Thus $l(J)=r(J)$, since the identity element 1 is a sum of primitive idempotents by the proof of Theorem 3. By (1), we have

$$
e r(J)=r(R(1-e)+J e) \neq 0
$$

for each nonzero idempotent $e$. Thus $l(r(J))$ does not contain any nonzero idempotent and $l(r(J))=J$ by Theorem 5 .

(4) Let $L$ be a nonzero left ideal. Take a finitely generated left subideal $L^{\prime} \neq 0$. $r\left(L^{\prime}\right) \subset I$ for some maximal right ideal $I$. Then

$$
L \supset L^{\prime}=l\left(r\left(L^{\prime}\right)\right) \supset l(I) \text {. }
$$

Thus $L$ contains a minimal left ideal $l(I)$. Similarly, each nonzero right ideal contains a minimal right ideal (or by the proof of Theorem 3 ).

3. PF-rings. $A$ ring $R$ is called right $\mathrm{PF}$ if every faithful right $R$-module is a generator in $\mathfrak{M}_{R}$. In this section we give characterizations of right PF-rings.

Let $C$ be defined as in Theorem 3 .

THEOREM 7. The following conditions on a ring $R$ are equivalent:

(1) $R$ is right $\mathrm{PF}$.

(2) $R$ is right self-injective and $l(I) \neq 0$ for each maximal right ideal $I$.

(3) $R$ is an injective cogenerator in $\mathfrak{M}_{R}$.

(4) $R / J$ is Artinian and $E(U)$ is projective for each simple right $R$-module $U$.

PROOF. $(2) \Leftrightarrow(3)$ by Theorem 1 .

(3) implies (4). $R / J$ is Artinian by Theorem 2. $E(U)$ is projective for each simple right $R$-module $U$ since $R$ is a cogenerator in $\mathfrak{M}_{R}$.

(4) implies (3). Let $U$ be a simple right $R$-module. Since $E(U)$ is indecomposable injective and projective, $R$ contains a copy of $E(U)$ by $[4$, Corollary 2.5]. Hence $R$ is a cogenerator in $\mathfrak{M}_{R}$. $R$ is right self-injective since $R$ is a 
cogenerator in $\mathfrak{M}_{R}$ and $R / J$ is Artinian by [4, Corollary 4.2].

(1) implies (3). $C$ is faithful since $C$ is a cogenerator in $\mathfrak{M}_{R}$. By assumption, $C$ is a generator in $\mathfrak{M}_{R}$, Hence $R$ is an injective cogenerator in $\mathfrak{M}_{R}$ by Theorem 3.

(3) implies (1). Let $M$ be a faithful right $R$-module. Then $M$ is a cogenerator in $\mathfrak{M}_{R}$ by the next lemma. Hence $M$ contains a copy of $C$, which is injective since $R / J$ is Artinian. Hence we have an epimorphism

$$
M \longrightarrow C \longrightarrow 0
$$

Thus $M$ is a generator in $\mathfrak{M}_{R}$, since $C$ is a generator in $\mathfrak{M}_{R}$ by Theorem 3 .

LEMMA 2. Lot $R$ be a cogenerator in $\mathfrak{M}_{R}$. Then the following conditions on a right $R$-module $M$ are equivalent:

(1) $M$ is faithful.

(2) $M$ is a cogenerator in $\mathfrak{M}_{R}$.

PROOF. (2) trivially implies (1).

(1) implies (2). Let $U$ be a simple right $R$-module. Note that every nonzero submodule of $E(U)$ contains $U$ since $E(U)^{\prime} \supset U$. Now, we may assume that $E(U) \subset R$ since $R$ is a cogenerator in $\mathfrak{M}_{R}$. Then $M U \neq 0$ since $M$ is faithful. Take $m \in M$ such that $m U \neq 0$. Then the map

$$
E(U) \longrightarrow m E(U) \subset M
$$

must be a monomorphism since $m U \neq 0$. Thus $M$ contains a copy of $E(U)$, thus completing the proof.

It may be interesting to compare our Theorem 7 with the following theorem, which was proved in G.Azumaya [1] or in Y.Utumi [10].

THEOREM. A ring $R$ is right $\mathrm{PF}$ if and only if $R$ is right self-injective and a finite direct sum of indecomposable right ideals each of which contains a minimal right ideal of $R$.

It is to be noted that our characterizations of (right) PF-rings are farreaching (see the next section). Note also that if $R$ is a right PF-ring then $r(l(I))=I$ for each right ideal $I$ of $R$.

4. Duality. Let us consider a problem to find out possible types of rings for which every finitely generated module is reflexive. In the following, we shall give a complete solution for this problem.

THEOREM 8. The following conditions on a ring $R$ are equivalent: 
(1) Every submodule and every quotient module of a reflexive right $R$-module is reflexive and every submodule and every quotient module of a reflexive left $R$-module is reflexive.

(2) Every finitely generated right, and every finitely generated left, $R$ module is reflexive.

(3) Every cyclic right, and every cyclic left, $R$-module is reflexive.

(4) $R$ is PF.

(5) $R$ is self-injective and $l(I) \neq 0$ for each maximal right ideal $I$.

(6) $R$ is an injective cogenerator both in $\mathfrak{M}_{R}$ and in ${ }_{R} \mathfrak{M}$.

ProOf. The implications $(1) \Rightarrow(2) \Rightarrow(3)$ are trivial.

(3) implies (4). $R$ is self-injective, each right ideal is a right annulet and each left ideal is a left annulet, by [6, Theorem 12]. Hence $R$ is PF by Theorem 7.

(4) implies (5). This implication is trivial by Theorem 7.

(5) implies (6). Note $r(L) \neq 0$ for each maximal left ideal $L$ by Theorem 6. Hence $R$ is an injective cogenerator both in $\mathfrak{M}_{R}$ and in ${ }_{R} \mathfrak{M}$.

(6) implies (1). Let

$$
0 \rightarrow A \rightarrow B \rightarrow B / A \rightarrow 0
$$

be an exact sequence of right (or left) $R$-modules with $B$ reflexive. Then we have the commutative exact diagram

$$
\begin{aligned}
0 \rightarrow A^{* *} & \rightarrow B^{* *} \rightarrow(B / A)^{* *} \rightarrow 0 \\
\delta_{A} \uparrow & \Downarrow \\
0 \rightarrow A & \rightarrow B
\end{aligned} \quad \begin{aligned}
& \uparrow \delta_{B / A} \\
&
\end{aligned}
$$

since $R$ is self-injective.

Now, $\delta_{B / A}$ is a monomorphism since $R$ is a cogenerator both in $\mathfrak{M}_{R}$ and in ${ }_{R} \mathfrak{M}$. Hence $\delta_{B / A}$ is an isomorphism. This, in turn, forces $\delta_{A}$ to be an isomorphism. This completes the proof of the theorem.

Let $R$ be a ring, and $A$ a right $R$-module. If $X$ is a subset of $A$ (resp. $\left.A^{*}\right)$ we denote its annihilator in $A^{*}$ (resp. $A$ ) by $l(X)$ (resp. $r(X)$ ). Note that, if $A_{0}$ is a submodule of $\left.A, r^{\prime} l\left(A_{0}\right)\right)=A_{0}$ if and only if $A / A_{0}$ is torsionless.

Now, recall the definition of $\delta_{A}: A \rightarrow A^{* *}$. $\delta_{A}$ is defined by the rule

$$
b \delta_{A}(a)=b a
$$

for all $b \in A^{*}, a \in A$.

THEOREM 9, Let $R$ be a ring characterized in Theorem 8 , and $A$ a 
right $R$-module. Then

(1) $r\left(l\left(A_{0}\right)\right)=A_{0}$ for every submodule $A_{0}$ of $A$.

(2) If $A$ is finitely generated (or reflexive), then $l\left(r\left(B_{0}\right)\right)=B_{0}$ for every submodule $B_{0}$ of $A^{*}$.

PROOF. $R$ is a cogenerator both in $\mathfrak{M}_{R}$ and in ${ }_{R} \mathfrak{M}$. We use this fact to show (1) and (2).

(1) $A / A_{0}$ is torsionless since $R$ is a cogenerator in $\mathfrak{M}_{R}$. Thus $r\left(l\left(A_{0}\right)\right)=A_{0}$.

(2) The inclusion $l\left(r\left(B_{0}\right)\right) \supset B_{0}$ is obvious. Since $A$ is finitely generated, $A$ is reflexive by Theorem 8. Hence each element of $A^{* *}$ is of the form $\delta_{A}(a)$ for some $a \in A$. Moreover, $A^{*} / B_{0}$ is torsionless since $R$ is a cogenerator in ${ }_{R} \mathfrak{M}$. Now, let $b \in A^{*}, b \notin B_{0}$. Then there exists an element $\delta_{A}(a)$ of $A^{* *}$ such that

$$
B_{0} \delta_{A}(a)=0, b \delta_{A}(a) \neq 0
$$

since $A^{*} / B_{0}$ is torsionless (see Proposition 1,(3)). Hence $B_{0} a=0, b a \neq 0$, or equivalently, $a \in r\left(B_{0}\right), b a \neq 0$. Hence $b \notin l\left(r\left(B_{0}\right)\right)$, which completes the proof of the theorem.

Theorem 9 is a generalization of a theorem of M.Hall, who considered the case $: R$ is a $Q F$-ring and $A$ is a finite free $R$-module.

In connection with the preceding theorem, we have the following

LEMMA 3. Let $R$ be a ring characterized in Theorem 8, and $A$ a infinite direct sum of right $R$-modules $A_{i}$. Then $A^{*}=\Pi A_{i}{ }^{*} \supset \sum A_{i}^{*} l\left(r\left(\sum A_{i}^{*}\right)\right)$ $\neq \sum A_{i}{ }^{*}$ and $A$ is not reflexive.

PRoOF. $r\left(\sum A_{i}^{*}\right)=0$ since each $A_{i}$ is torsionless. Hence

$$
l\left(r\left(\sum A_{i}^{*}\right)\right)=A^{*}=\Pi A_{i}^{*} \neq \sum A_{i}^{*}
$$

Therefore $A$ is not reflexive by Theorem 9 .

We can now prove the following

THEOREM 10. The following conditions on a ring $R$ are equivalent:

(1) $R$ is $\mathrm{PF}$ and the Jacobson radical $J$ of $R$ is nilpotent.

(2) $R$ is QF.

ProOF. (1) implies (2). $\left(J^{i} / J^{i+1}\right)_{R}$ is reflexive by Theorem 8 . Since $R / J$ 
is Artinian, $J^{i} / J^{i+1}$ is a direct sum of simple submodules. By Lemma 3 , $J^{i} / J^{i+1}$ must be a finite direct sum of simple submodules and hence $J^{i} / J^{i+1}$ has a composition series for each $i$. Thus $R_{R}$ has a composition series since $J$ is nilpotent.

(2) trivially implies (1) by Theorem 8.

On duality over right PF-rings, we have the following

PROPOSITION 4. The following conditions on a ring $R$ are equivalent:

(1) If $B$ is a left $R$-module such that $\delta_{B}$ is an epimorphism, and if $A$ is any quotient module of $B$, then $\delta_{A}$ is an epimorp.nism. Moreover, every right $R$-module is torsionless.

(2) $\delta_{R / L}$ is an epimorphism for every left ideal $L$ of $R$, and $R / I$ is torsionless for every right ideal $I$ of $R$.

(3) $R$ is an injective cogenerator in $\mathfrak{M}_{R}$.

(4) $R$ is right $\mathrm{PF}$.

PROOF. (1) triviaily implies (2).

(2) implies (3). Let $I$ be a right ideal of $R$. Since $R / I$ is torsionless, $I=r(L)$ for some left ideal $L$ of $R$ by [6, Theorem 1]. By [6, Proposition 7], $\operatorname{Ext}_{R}^{1}(R / I, R)=0$ since $\delta_{R / L}$ is an epimorphism. Thus $R$ is right self-injective. Moreover $\left.r^{\prime} l(I)\right)=I$ for each right ideal $I$ since $R / I$ is torsionless. Hence $R$ is an injective cogenerator in $\mathfrak{M}_{R}$ by Theorem 1 .

$(3) \Leftrightarrow(4)$ by Theorem 7 .

(3) implies (1). Every right $R$-module is torsionless since $R$ is a cogenerator in $\mathfrak{M}_{R}$. Next, let $A$ be a quotient module of a left $R$-module $B$ for which $\delta_{B}$ is an epimorphism. Then we have an exact sequence of right $R$-modules $0 \rightarrow A^{*} \rightarrow B^{*}$. From this we get the following commutative exact diagram

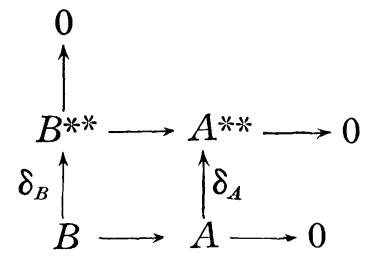

since $R$ is right self-injective. Thus $\delta_{A}$ is an epimorphism.

5. Some open problems. It is an open question whether a right PF-ring is left PF or not. If a right PF-ring is necessarily left self-injective, then the above question is answered affirmatively from what we have seen.

We have seen that, in a right PF-ring, every right ideal is a right annulet. Is every left ideal a left annulet in a right PF-ring? In this connection, we have seen that finitely generated left ideals, maximal left ideals, 
and $J$ are left annulets in a right PF-ring.

\section{REFERENCES}

[1] G. Azumaya, Completely faithful modules and self-injective rings, Nagoya Math. Journ., 27(1966), 697-708.

[21 H. BAss, Finitistic dimension and a homological generalization of semi-primary rings, Trans. Amer. Math. Soc., 95(1960), 466-488.

[3] C. FAITH AND Y. UTUMI, Quasi injective modules and their endomorphism rings, Arch. Math., 15(1964), 166-174.

[4] C.FAITH AND E.A.WALKER, Direct sum representations of injective modules, Journ. Algebra, 5(1967), 203-221.

[5] M. IKEDA AND T.NAKAYAMA, On some characteristic properties of quasi-Frobenius and regular rings, Proc. Amer. Math. Soc., 5(1954), 15-19.

[6] T.KATO, Duality of cyclic modules, Tôhoku Math. Journ. 19(1967), 349-356.

[7] J.LAMBEK, On the ring of quotients of a Noetherian ring, Canad. Math. Bull., 8 (1965), 279-290.

[8] B.L. Osofsky, A generalization of quasi-Frobenius rings, Journ. Algebra, 4(1966), 373-387.

[9] Y.UTUMI, On continuous rings and self-injective rings, Trans. Amer. Math. Soc., 118(1965), 158-173.

[10] Y.UTUMI, Self-injective rings, Journ. Algebra, 6(1967), 56-64.

\section{MATHEMATICAL INSTITUTE}

TÔHOKU UNIVERSITY

SENDAI, JAPAN. 